\title{
RATIONAL APPROXIMATIONS TO L-S CATEGORY AND A CONJECTURE OF GANEA
}

\author{
BARRY JESSUP
}

\begin{abstract}
The rational version of Ganea's conjecture for L-S category, namely that $\operatorname{cat}\left(S \times \Sigma^{k}\right)=\operatorname{cat}(S)+1$, if $S$ is a rational space and $\Sigma^{k}$ denotes the $k$-sphere, is still open. Recently, a module type approximation to cat $(S)$, $M \operatorname{cat}(S)$, was introduced by Halperin and Lemaire. We have previously shown that $M$ cat satisfies Ganea's conjecture. Here we show that for $(r-1)$ connected $S$, if $M$ cat $(S)$ is at least $\operatorname{dim} S / 2 r$, then $M$ cat $(S)=\operatorname{cat}(S)$. This yields Ganea's conjecture for these spaces. We also extend other properties of $M$ cat, previously unknown for cat, to these spaces.
\end{abstract}

\section{INTRODUCTION}

Let $S$ be a pointed, simply connected space with the homotopy type of a CW complex of finite type. The Lusternik-Schnirelmann category of $S$, cat $(S)$, is the least $m$ such that $S$ may be covered by $m+1$ open sets, each contractible in $S$ [Lu-Sc].

L-S category is a subtle invariant which is usually difficult to compute. (The survey article by James [Ja] is an excellent reference.) Well-known bounds for $\operatorname{cat}(S)$ are

$$
\text { cup length } H^{*}(S ; R) \leq \operatorname{cat}(S) \leq \frac{\operatorname{dim} S}{r}
$$

if $S$ is $(r-1)$ connected, but these determine cat $(S)$ only in simple cases. The behaviour of cat on products is complicated: it is straightforward that

$$
\operatorname{cat}(S \times T) \leq \operatorname{cat}(S)+\operatorname{cat}(T),
$$

and strict inequality is possible. The only known examples of this, however, occur when both $S$ and $T$ have coprime torsion. Ganea $\left[\mathrm{Ga}_{1}\right]$ generalized examples of Fox [Fo] and conjectured $\left[\mathrm{Ga}_{2}\right]$ that if one factor, say a $k$-sphere, had no torsion, then equality should hold. This has become known as

Ganea's Conjecture. If $S$ is a finite complex and $\Sigma^{k}$ denotes the $k$-sphere then

$$
\operatorname{cat}\left(S \times \Sigma^{k}\right)=\operatorname{cat}(S)+1, \quad k \geq 1 .
$$

Received by the editors May 5, 1988.

1980 Mathematics Subject Classification (1985 Revision). Primary 55M30, 55P60, 55P62.

Key words and phrases. Lusternik-Schnirelmann category, minimal models, rational homotopy. 
The truth of this conjecture is unknown in general. Singhof [Si] has shown that if $M$ is an $(r-1)$ connected piecewise-linear $n$-manifold $(n \geq 4)$ with

$$
\operatorname{cat}(M) \geq \frac{n+r+k+3}{2 r}-1
$$

then Ganea's conjecture is true for $M$.

Even if we restrict our attention to the rational case, thereby elimating all torsion from both factors, Ganea's conjecture is still open.

If $S_{\mathrm{Q}}$ denotes the localization of $S$ at the rationals, Felix and Halperin [Fe-Ha] have described cat $\left(S_{\mathbf{Q}}\right) \equiv \operatorname{cat}_{0}(S)$, the rational category of $S$ via a Sullivan minimal model for $S$. Later, Halperin and Lemaire [Ha-Le] introduced $M$ cat $_{0}(S)$, another homotopy invariant which is a "module-type" approximation to $\operatorname{cat}_{0}(S)$ and which satisfies

$$
M \operatorname{cat}_{0}(S) \leq \operatorname{cat}_{0}(S) .
$$

At present, the author knows of no example where $M$ cat $_{0}(S) \neq \operatorname{cat}_{0}(S)$. This is particularly interesting in view of previous work [J] where we have proven Ganea's conjecture for $M$ cat $_{0}$, namely, that

$$
M \mathrm{cat}_{0}\left(S \times \Sigma^{k}\right)=M \mathrm{cat}_{0}(S)+1 .
$$

In the cases when $M \mathrm{cat}_{0}(S)=\operatorname{cat}_{0}(S)$, the inequalities

$$
\operatorname{cat}_{0}(S)+1=M \operatorname{cat}_{0}(S)+1=M \operatorname{cat}_{0}\left(S \times \Sigma^{k}\right) \leq \operatorname{cat}_{0}\left(S \times \Sigma^{k}\right) \leq \operatorname{cat}_{0}(S)+1
$$

show that the rational version of Ganea's conjecture is true for $S$; that is

$$
\text { cat }_{0}\left(S \times \Sigma^{k}\right)=\text { cat }_{0}(S)+1 \text {. }
$$

We have already been able to show that $M \operatorname{cat}_{0}(S)=\operatorname{cat}_{0}(S)$ when $S=G / H$ is a homogeneous space and $G$ and $H$ are compact, simply connected Lie groups with $\operatorname{rank} G-\operatorname{rank} H=0$ or 1 [J, Theorem $\left.5.1^{\prime}\right]$. The main result of this paper is

Theorem 1. Let $S$ be $(r-1)$ connected and suppose that $H^{>n}(S ; \mathbf{Q})=0$. If $M \mathrm{cat}_{0}(S) \geq n / 2 r$, then $M \mathrm{cat}_{0}(S)=\operatorname{cat}_{0}(S)$,

and the

Corollary 1. Let $S$ be as in Theorem 1. Then

$$
\operatorname{cat}_{0}\left(S \times \Sigma^{k}\right)=\operatorname{cat}(S)+1 .
$$

Remark. Since for $(r-1)$ connected $S$ we have $M$ cat $_{0}(S) \leq n / r$, this theorem applies to spaces where $M$ cat $_{0}(S)$ lies in the upper half of its possible range.

It is important to note that our result is not the rational version of Singhof's theorem. The bound in the latter depends on the dimension of the sphere, while ours does not. For a fixed $S$ satisfying the hypotheses, we can thus deduce the veracity of Ganea's conjecture for all the spheres $\Sigma^{k}, k \geq 1$.

In [J] we proved a theorem more general than Ganea's conjecture for $M$ cat $_{0}$. If $e_{0}(T) \equiv e\left(T_{\mathrm{Q}}\right)$ denotes Toomer's invariant for $T_{\mathrm{Q}}$ (defined in terms of the 
Milnor-Moore spectral sequence [To]), we proved

[J, Theorem 4.1]. If $S$ and $T$ are simply connected $C W$ complexes of finite type, then

$$
M \operatorname{cat}_{0}(S)+e_{0}(T) \leq M \operatorname{cat}_{0}(S \times T) .
$$

Applying Theorem 1, we have the

Corollary 2. Let $S$ be as in Theorem 1. Then

$$
\operatorname{cat}_{0}(S \times T) \geq \operatorname{cat}_{0}(S)+e_{0}(T) .
$$

In particular, if $T$ is formal or coformal (so that $e_{0}(T)=$ cat $_{0}(T)$ ), then

$$
\text { cat }_{0}(S \times T)=\text { cat }_{0}(S)+\operatorname{cat}_{0}(T) .
$$

(Note that Corollary 1 is a special case of Corollary 2.)

The characterization of $\mathrm{cat}_{0}(S)$ and $M \mathrm{cat}_{0}(S)$ by retractions of commutative graded differential algebras (CGDAs) over the rationals allows one to define L-S type homotopy invariants cat ${ }_{K}(S)$ and $M$ cat $_{K}(S)$ for any $K \supset \mathbf{Q}$. (Indeed, even more is possible. See [Ha-Le].) It is elementary that cat $_{K}(S) \leq$ cat $_{0}(S)$ but it is unknown whether equality holds in general. However, we have shown [J, Theorem 3.2]) that $M \mathrm{cat}_{K}(S)=M \mathrm{cat}_{0}(S)$ for any $K \supset \mathbf{Q}$. In view of Theorem 1 and the straightforward inequality $M \operatorname{cat}_{K}(S) \leq \operatorname{cat}_{K}(S)$, we have

Corollary 3. Let $K \supset \mathbf{Q}$ be any field extension. If $S$ is as in Theorem 1 , then cat $_{K}(S)=$ cat $_{0}(S)$.

This paper is organized as follows. In the next section, we recall the results from rational homotopy we will need and then define rational category, and its generalizations, for field extensions $K \supset \mathbf{Q}$. Theorem 1 is proved in $\S 3$.

\section{RATIONAL CATEGORY}

For the homotopy theory of commutative graded differential algebras (CGDAs), the reader is referred to fundamental treatise in the subject [Su] by Sullivan and for notation and complete details to [Ha]. An excellent summary of the material appears in [Fe-Ha] and the interesting article by Avramov and Halperin [Av-Ha] is recommended. Unless otherwise stated, we work over the rationals $\mathbf{Q}$.

For our purposes here, we recall the following facts. Let $(A, d) \stackrel{\varphi}{\rightarrow}(B, d)$ be a morphism between two CGDAs with $H^{0}(A)=H^{0}(B)=\mathbf{Q}$. Then a minimal Sullivan model of $\varphi$ is a diagram

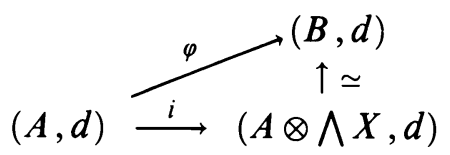

where the vertical arrow is a weak equivalence (i.e. an isomorphism on cohomology) and $(A, d) \rightarrow(A \otimes \wedge X, d)$ is a $K S$ extension as defined in [Ha]. In particular, $i(a)=a \otimes 1$ and $X$ has a vector space basis $\left\{x_{\alpha} \mid \alpha \in I\right\}$, called a 
$K S$ basis, such that $d x_{\alpha} \in A \otimes \bigwedge X_{<\alpha}$ and $\alpha<\beta \Rightarrow \operatorname{deg} x_{\alpha} \leq \operatorname{deg} X_{\beta}$. Here, $X_{<\alpha}$ denotes $\operatorname{span}\left\{x_{\beta} \mid \beta<\alpha\right\}$. This diagram is determined up to isomorphism by $\varphi$ and we say that $i$ represents $\varphi$.

We remark that if $\varphi^{*}$ is surjective in all degrees less than $n$ and injective in all degrees less than $n+1$, then $X=X^{\geq n}$ [Ha, 6.16, Remark 2].

The connection to topology is that Sullivan defined a functor $A$ which associates to every space $S$ a CDGA over $\mathbf{Q},(A(S), d)$ (consisting of the rational differential forms on the singular simplices of $S$ ) which calculates the cchomology of $S$ with rational coefficients: $H^{*}(A(S), d) \cong H^{*}(S ; \mathbf{Q})$. (See [Ha, 15.6 and 14.18] for example.) The minimal Sullivan model of $(\mathbf{Q}, 0) \rightarrow(A(S), d)$ is of the form $(\mathbf{Q}, 0) \rightarrow(\bigwedge X, d) \stackrel{\cong}{\rightrightarrows}(A(S), d)$ and $(\bigwedge X, d)$ is called the Sullivan minimal model of $S$. Sullivan [Su] showed that $(\bigwedge X, d)$ carries the rational homotopy type of $S$. In particular, $X^{*} \cong \operatorname{Hom}_{\mathbf{z}}\left(\pi_{*}(S), \mathbf{Q}\right)$.

Let $m$ be a positive integer and $(\bigwedge X, d)$ be a Sullivan minimal model for a space $S$. Let

$$
\left(\bigwedge_{j \downarrow}(\bigwedge x, d) \underset{j \downarrow}{\stackrel{\pi}{\rightarrow}}\left(\bigwedge X / \bigwedge^{>m} X, d\right)\right.
$$

be a minimal Sullivan model for the projection $\pi$. Theorem 4.7 of [Fe-Ha] states that $\operatorname{cat}_{0}(S)$ is the least $m$ such that the inclusion $j$ above admits a CGDA retraction

$$
(\bigwedge X \otimes \bigwedge Y, d) \stackrel{r}{\rightarrow}(\bigwedge X, d)
$$

That is, $r$ is a morphism of CGDAs which satisfies $r \circ j=\mathrm{id}_{\wedge X}$.

If $K \supset \mathbf{Q}$ is any field extension, then $\operatorname{cat}_{K}(S)$ is defined as cat $_{0}(\bigwedge X \otimes K, d)$, where in diagram (3.1) we work over $K$ and require $r$ to be a retraction of CDG $K$-algebras. By tensoring the diagram (3.1) with $K$ and noting that $\Lambda X \otimes / \bigwedge^{>m} X \otimes K \cong\left(\bigwedge X / \bigwedge^{>m} X\right) \otimes K$, we see that cat $_{K}(S) \leq$ cat $_{0}(S)$.

The definitions of $M$ cat $_{0}(S)$ and $M$ cat $_{K}(S)$ are similar. First note that both $(\bigwedge X, d)$ and $(\bigwedge X \otimes \bigwedge Y, d)$ are $(\bigwedge X, d)$ differential modules. Then, $M$ cat $_{0}(S)$ is the least integer $m$ such that the inclusion $j$ in (3.1) admits a retraction $(\bigwedge X, d) \stackrel{r}{\rightarrow}(\bigwedge X \otimes \bigwedge Y, d)$ of $(\bigwedge X, d)$ differential modules.

The definition of $M \mathrm{cat}_{K}(S)$ is analogous to that of $\mathrm{cat}_{K}(S)$, except that we require only that the retraction respect the $(\bigwedge X \otimes K, d)$ differential module structures. Again, it is elementary that $M$ cat $_{K}(S) \leq M$ cat $_{0}(S)$.

It is interesting to note that Toomer's invariant $e_{0}(S)$ may be defined as $\operatorname{cat}_{0}(S) \& M$ cat $_{0}(S)$ have been. Indeed, $e_{0}(S)$ is the least $m$ such that $j$ in (3.1) admits a retraction of linear differential spaces.

\section{Proof of Theorem 1}

It is clear from the definitions that $M$ cat $_{0}(S) \leq \operatorname{cat}_{0}(S)$. To prove equality, it suffices therefore to show that $M$ cat $_{0}(S) \geq$ cat $_{0}(S)$. So suppose $n=\operatorname{dim} S$ and that $S$ is $(r-1)$ connected. Let $(\bigwedge X, d)$ be a Sullivan minimal model for 
$S$. Set $m=M \operatorname{cat}_{0}(S)$ and let $(\bigwedge X, d) \rightarrow(\bigwedge X \otimes \bigwedge Y, d) \stackrel{\simeq}{\rightarrow}\left(\bigwedge X / \bigwedge^{>m} X, d\right)$ be a minimal Sullivan model for the projection $(\Lambda X, d) \stackrel{\pi}{\rightarrow}\left(\Lambda X / \Lambda^{>m} X, d\right)$. The retraction $(\bigwedge X \otimes \Lambda Y, d) \stackrel{r}{\rightarrow}(\bigwedge X, d)$ guaranteed by $M$ cat $_{0}(S)=m$ ensures that $\pi^{*}: H(\bigwedge X, d) \rightarrow H\left(\bigwedge X / \bigwedge^{>m} X, d\right)$ is injective. Moreover, $\pi^{*}$ is surjective in all degrees less than $(m+1) r-1$. To see this, note that for any cocycle $z \in \Lambda X / \Lambda^{>m} X$ which is not the image of a cocycle in $\Lambda X$, there is $a \in \Lambda X$ with $\pi(a)=z$ and $0 \neq d a \in \Lambda^{>m} X$. As $S$ is $(r-1)$ connected, $X=X^{\geq r}$ and so $\operatorname{deg} z=\operatorname{deg} a \geq(m+1) r-1$. By our earlier remark, these two statements about $\pi^{*}$ imply that $Y=Y^{\geq(m+1) r-1}$.

Now let $\left\{y_{\alpha} \mid \alpha \in I\right\}$ be a $K S$ basis for $(\bigwedge X \otimes \bigwedge Y, d)$ and let $Y_{<\alpha}=$ $\operatorname{span}\left\{y_{\beta} \mid \beta<\alpha\right\}$. Then we know that

$$
d y_{\alpha}=a_{\alpha}+\sum_{\beta<\alpha} a_{\alpha \beta} y_{\beta}+t_{\alpha}
$$

where $a_{\alpha}, a_{\alpha \beta} \in \Lambda^{+} X, y_{\beta} \in Y_{<\alpha}$ and $t_{\alpha} \in \Lambda X \otimes \Lambda^{\geq 2} Y_{<\alpha}$.

We will construct the required CGDA retraction $(\bigwedge X \otimes \Lambda Y, d) \stackrel{\bar{r}}{\rightarrow}(\bigwedge X, d)$ by induction on $\alpha$. The induction hypotheses will guarantee that $\bar{r}$ exists on $\left(\bigwedge X \otimes \wedge Y_{<\alpha}, d\right)$, and we will show how to extend it to $\left(\bigwedge X \otimes \wedge Y_{<\alpha}, d\right)$.

Define $\bar{r}(a)=a$ for all $a \in \Lambda X$. The induction hypothesis $\alpha$ consists of two parts:

$$
\begin{aligned}
& \alpha(A): \bar{r}\left(y_{\beta}\right)=r\left(y_{\beta}\right) \text { if } \operatorname{deg}\left(y_{\beta}\right)<n \text { and } \beta<\alpha, \\
& \alpha(B): \bar{r}\left(d y_{\beta}\right)=d \bar{r}\left(y_{\beta}\right) \text { if } \beta<\alpha .
\end{aligned}
$$

To begin the induction, suppose that 0 is the first element of $I$. Then $d y_{0}=0$ as $y_{0}$ arises from the cokernel $\pi^{*}\left(H(\Lambda X, d) / H\left(\Lambda X / \Lambda^{>m} X, d\right)\right)$. Set $\bar{r}\left(y_{0}\right)=r\left(y_{0}\right)$. Then $1(A)$ and $1(B)$ are satisfied.

Now assume the induction hypothesis for $\alpha>1$. We consider separately the two cases $\operatorname{deg}\left(y_{\alpha}\right)<$ or $\geq n$.

Case 1. $\operatorname{deg}\left(y_{\alpha}\right)<n$. In this case $t_{\alpha}$, the "nonlinear" part of $d y_{\alpha}$ is zero for degree reasons. Indeed, if $t_{\alpha} \neq 0$, then $\operatorname{deg}\left(y_{\alpha}\right)=\operatorname{deg}\left(t_{\alpha}\right)-1 \geq 2$. $[r(m+1)-1]-1 \geq 2 r(n / 2 r+1)-3$ which is at least $n$ since $r \geq 2$ and $m \geq n / 2 r$. (Actually, we need only assume $m \geq(n+3) / 2 r-1)$.

Moreover, since $\operatorname{deg}\left(a_{\alpha \beta}\right) \geq r \geq 2$, we know as well that $\operatorname{deg}\left(y_{\beta}\right)<n$ for all $y_{\beta}$ appearing in the expression for $d y_{\alpha}$. hence, by $\alpha(A)$, we may conclude that $\bar{r}\left(y_{\beta}\right)=r\left(y_{\beta}\right)$ for all such $y_{\beta}$. Together with the fact that $r$ is $\bigwedge X$-linear, this implies that $\bar{r}\left(d y_{\alpha}\right)=r\left(d y_{\alpha}\right)$, and so we may set $\bar{r}\left(y_{\alpha}\right)=r\left(y_{\alpha}\right)$. Thus $[\alpha+1](A)$ and $[\alpha+1](B)$ are satisfied.

It remains to consider

Case 2. $\operatorname{deg}\left(y_{\alpha}\right) \geq n$. In this case, $\operatorname{deg}\left(\bar{r}\left(d y_{\alpha}\right)\right) \geq n+1$. So, as $H^{>n}(\bigwedge X, d)=$ $0, \bar{r}\left(d y_{\alpha}\right)$ is exact, say $\bar{r}\left(d y_{\alpha}\right)=d b_{\alpha}, b_{\alpha} \in \Lambda X$. Define $\bar{r}\left(y_{\alpha}\right)=b_{\alpha}$. Then $[\alpha+1](B)$ is satisfied and the induction and the proof are complete.

Note added in proof. Since the original submission of this article, K. Hess has proved $\mathrm{cat}_{0}(S)=M \mathrm{cat}_{0}(S)$ for all simply connected spaces. 


\section{BIBLIOGRAPHY}

[Av-Ha] L. Avramov and S. Halperin, Through the looking glass: a dictionary between rational homotopy theory and local algebra, Algebra, Algebraic Topology and their Interactions, Lecture Notes in Math., vol 1183, Springer-Verlag, 1986, pp. 3-27.

[Fe-Ha] Y. Felix and S. Halperin, Rational L.-S. category and its applications, Trans. Amer. Math. Soc. 273 (1982), 1-37.

[Fo] R. H. Fox, On the Lusterik-Schnirelmann category, Ann. of Math. 42 (1941), 333-370.

$\left[\mathrm{Ga}_{1}\right]$ T. Ganea, Lusternik-Schnirelmann category and cocategory, Proc. London Math. Soc. (3) 10 (1960), 832-839.

$\left[\mathrm{Ga}_{2}\right]$ _ - Some problems on numerical homotopy invariants, Lecture Notes in Math., vol. 249, Springer, 1971, pp. 23-30.

[Ha-Le] S. Halperin and J.-M. Lemaire, Notions of category in differential algebra, Lecture Notes in Math., vol. 1318, Springer, 1988, pp. 138-154.

[Ha] S. Halperin, Lectures on minimal models, Mémoires de la S. M. F. 9-10 (1983).

[J] B. Jessup, Rational Lusternik-Schnirelmann category, fibrations and a conjecture of Ganea, Preprint.

[Ja] I. M. James, On category, in the sense of Lusternik-Schnirelmann, Topology 17 (1978), 331348.

[Lu-Sc] L. Lusternik and L. Schnirelmann, Méthodes topologiques dans les problèmes variationnels, Hermann, Paris, 1934.

[Si] W. Singhof, Minimal coverings of manifolds with balls, Manuscripta Math. 29 (1979), 385-415.

[Su] Dennis Sullivan, Infinitesimal computations in topology, Inst. Hautes Etudes Sci. Publ. Math. 47 (1978), 269-331.

[To] G. H. Toomer, Lusternik-Schnirelmann category and the Moore spectral sequence, Math. Z. 138 (1974), 175-180.

[Wh] G. W. Whitehead, The homology suspension, Colloque de Topologie Algébrique tenue à Louvain, 1956, pp. 89-95.

Department of Mathematics, York University, 4700 Keele Street, North York, ONtario, CanAda M3K 1 P3

Current address: Department of Mathematics, University of Ottawa, 585 King Edward, Ottawa, Ontario, Canada KIN 6N5 (VOMATHS @ UOTTOWA. BITNET) 